\title{
The Detailed Evolution of Carbon Spheres by Hydrothermal Method
}

\author{
Trevor Mwenya, ${ }^{1}$ Huiyang Fan, ${ }^{1}$ Han Dai, ${ }^{1}$ and Meicheng $\mathrm{Li}^{1,2}$ \\ ${ }^{1}$ State Key Laboratory of Alternate Electrical Power System with Renewable Energy Sources, North China Electric Power University, \\ Beijing 102206, China \\ ${ }^{2}$ Chongqing Materials Research Institute, Chongqing 400707, China
}

Correspondence should be addressed to Meicheng Li; mcli@ncepu.edu.cn

Received 23 December 2015; Accepted 15 February 2016

Academic Editor: Indrajit Shown

Copyright (C) 2016 Trevor Mwenya et al. This is an open access article distributed under the Creative Commons Attribution License, which permits unrestricted use, distribution, and reproduction in any medium, provided the original work is properly cited.

\begin{abstract}
Carbon spheres (CSs) can be synthesized easily by hydrothermal method using various solutions and a lot of mechanisms have been employed to explain their formation. In our work, some special phenomena such as the uniform size and surface corruption have been found as the reaction time increased. However, less attention has been focused on the detailed evolution phenomena of CSs. In order to understand these special phenomena well, classical nucleation theory was employed to study the reaction dynamics of CSs during the evolution processes. This work not only deeply reveals the evolution mechanism of CSs, but also opens a possible way for the control of size and morphologies of CSs through hydrothermal methods.
\end{abstract}

\section{Introduction}

Spherical carbon materials have received a great deal of interest in recent years for their unique physical and chemical properties such as controllable sizes, morphology, good adsorption performance, minimal surface energies, and mechanical properties [1-6]. Because of the advantages of carbon materials with microsizes, CSs are used in several applications such as lithium batteries [7-10], catalyst support [11-14], and and drug delivery [15-18]. Different methods have been used to synthesize carbon spheres such as the templating method $[19,20]$, pyrolysis of carbon sources [21, $22]$, chemical vapor disposition [23, 24], and hydrothermal methods $[25,26]$. Due to the economic, efficient, and "green" properties of hydrothermal method, CSs synthesized by this method have gained great interest recently [27]. Through hydrothermal method, controllable shape and size of CSs can be easily obtained in solution conditions [28]. The formation mechanisms of CSs in solution conditions have been studied by a lot of researches. However, controversies still exist in the growth process of CSs.

In this work, a series of experiments are conducted to study the mechanism behind the changes in the morphologies and size of CSs by hydrothermal method. In our experiment, with respect to the reaction time and the special morphologies of CSs, the evolution of CSs was divided into four stages: nucleation, carbonization, isokinetic growth, and corruption. As expected, significant size changes and surface corruption are observed during the growth of CSs. In order to understand the growth process of CSs in solutions well, classical nucleation theory [29], the solution free energy, and the Gibbs free energy of CSs are employed.

\section{Experiment}

The CSs were fabricated via hydrothermal method by reacting poly(vinylpyrrolidone) (PVP-K30, MW $=30,000)$ with ethylene glycol (EG, 99\%) in the presence of undiluted hydrochloric acid $(\mathrm{HCl}, 36 \% \sim 38 \%)$. Firstly, a $15 \mathrm{~mL}$ EG solution with $0.2 \mathrm{~g}$ of PVP was prepared. Then, drop by drop, $5 \mathrm{~mL}$ of $\mathrm{HCl}$ was added to this mixture. The mixed PVP/EG/HCl solution was further stirred for several minutes until it became uniform. In the typical synthesis, all the solutions were under constant stirring. This solution was then transferred into a $25 \mathrm{~mL}$ Teflon-lined autoclave tube and autoclaved in the drying tunnel at $160^{\circ} \mathrm{C}$ for $5-22 \mathrm{~h}$. After reaction, the solution was cooled down to room temperature. The black precipitate was collected and sequentially washed with deionized water. 
TABLE 1: The overall compositions of CSs in our experiments.

\begin{tabular}{lccc}
\hline \multirow{2}{*}{ CSs } & $\mathrm{C}(\%)$ & $\mathrm{H}(\%)$ & $\mathrm{O}(\%)$ \\
& 80.84 & 8.5 & 10.66 \\
\hline
\end{tabular}

TABLE 2: The overall compositions of CSs in other experiments.

\begin{tabular}{lccc}
\hline \multirow{2}{*}{ CSs } & $\mathrm{C}(\%)$ & $\mathrm{H}(\%)$ & $\mathrm{O}(\%)$ \\
& 66.24 & 3.73 & 30.03 \\
\hline
\end{tabular}

Then, the precipitate was dried at $80^{\circ} \mathrm{C}$ for $6 \mathrm{~h}$. Finally, brown solid samples (CSs) were obtained. Elemental analysis (C, H, and $\mathrm{O}$ ) of the samples was carried out on an EA 3000 analyzer (EuroVector, Milan, Italy). Scanning electron microscope (SEM) (FEI Quanta 200F, Hillsboro, OR, USA) was used to characterize the morphologies and size distributions of the samples.

\section{Results and Discussion}

A new reaction system with low reaction rate (duration about 22 hours in total) is adopted, which is better to show the details of reaction process in our study. In order to determine the reaction products, elemental analysis was carried out. The major content of brown solid samples obtained in our experiment is carbon. The composition of the CSs obtained after $6 \mathrm{~h}$ of reaction time is shown in Table 1 . It can be seen from Table 1 that the carbon content in CSs from our experiment is very high with a yield of $80 \%$. Table 2 shows the carbon content of CSs prepared using the traditional hydrothermal method with glucose as a carbon source which has a yield of about $67 \%$ [30]. Compared to the traditional way, the new reaction system provides a more efficient way for obtaining CSs with higher carbonization.

With reaction time as a control parameter, four types of reaction products have been observed in our experiments, as shown in Figure 1. At the reaction time of $5 \mathrm{~h}$, a lot of small particles (diameter is nearly $100 \mathrm{~nm}$ ) are agglomerated together, which can be clearly observed in Figure 1(a). After $6 \mathrm{~h}$, particles with regular spherical shape and smooth surfaces are formed. Of particular interest, these particles have different diameters. The diameters range from $150 \mathrm{~nm}$ to $1.4 \mu \mathrm{m}$. As the reaction proceeds further to the duration of $9 \mathrm{~h}$, all of the spheres have very similar morphology and particle sizes (about $2.7 \mu \mathrm{m}$ ), as shown in Figure 1(c). The ultrasimilar particle size and smooth surface of these particles were maintained for 13 hours. After the whole reaction time of around $22 \mathrm{~h}$, abnormal corruption occurs on the surface of some spheres as shown in Figure 1(d). In this stage, the size of CSs is relatively uniform and the average diameter of the spheres is $3.8 \mu \mathrm{m}$. However, many crater-shaped holes are formed on the particle surface which make it look as rough as the lunar surface. In addition, some irregular particles are formed around the larger and regular sphere particles.

Obviously, CSs exhibit various morphologies during their evolution process. According to the four types of reaction products, the growth of CSs was divided into four stages: nucleation, carbonization, isokinetic growth, and corruption.
Based on the classical nucleation theory, the nucleation of CSs is mainly attributed to the solution free energy and the Gibbs free energy of CSs. The changes of these two types of energy determine the development of CSs' growth process. In detail, the free energy of the solution is given by the following expression [31]:

$$
\Delta G_{s}=\Delta G_{0}+R T \cdot \ln J,
$$

where $\Delta G_{s}$ is the solution free energy, $\Delta G_{0}$ is the standard Gibbs free energy, $R$ is gas constant, $T$ is the temperature, and $J$ is response entropy. $J$ is proportional to the solution concentration. From (1), we know that $\Delta G_{s}$ decreases with decrease in solution concentration. The relationship between the Gibbs free energy of CSs and the radius of CSs is given by the equation [32]

$$
\Delta G_{c}=4 \pi \sigma\left(r^{2}-\frac{2 r^{3}}{3 r_{1}}\right)
$$

where $\Delta G_{c}$ is the work of formation of CSs, $r$ is the radius of CSs, $\sigma$ is the interfacial tension, and $r_{1}$ is the critical nucleus radius of CSs. Inferred from (2), as the CSs grow in size, $\Delta G_{c}$ increases at first and then starts to decrease.

Therefore, the detailed evolution process of CSs can be well understood by the energy changes in the CSs and the solution. Schematic diagrams and SEM images of the four stages of the evolution process of CSs are given in Figures 2 and 3 , respectively. At the initial stage, PVP in the solution is decomposed into polyaromatic hydrocarbons (PAHs) which are the precursors of carbon nuclei under high temperature and high pressure, as shown in Figures 2(a) and 2(b) [27]. Figure 3(a) shows the SEM image corresponding to the initial stage. In this stage, the concentration of PAHs is very high and the size of the nuclei is very small, resulting in high $\Delta G_{s}$ and low $\Delta G_{c} . \Delta G_{s}$ greatly exceeds $\Delta G_{c}$ which leads to fast and constant nucleation. Thereafter, the aggregated nuclei are oxidized into amorphous carbon. As the reaction proceeds to the second stage, the size distribution of CSs is wide, as shown in Figures 2(c) and 3(b). Subsequently, all the CSs keep growing and gradually the sizes of CSs become uniform after 3 hours as shown in Figures 2(d) and 3(c). The exhibition of different particle sizes of CSs can be explained by the different nuclei time of the CSs. However, the abnormal uniform sizes of CSs cannot be well understood by the common sense. Since the change in size of CSs is also based on their Gibbs free energy and solution free energy, the size changes of CSs in this stage can also be analyzed by the changes of $\Delta G_{s}$ and $\Delta G_{c}$.

Based on (1) and (2), it is clear that the energy gap between $\Delta G_{s}$ and $\Delta G_{c}$ of bigger CSs is lower than that of smaller CSs. This indicates that the growth rates of CSs with small particle sizes are much higher than those of the big ones. Thus, small CSs grow faster than the big ones in the solution. Eventually, all the CSs reach a similar size. After a very long reaction time $(14 \mathrm{~h})$, the growth of the CSs nearly stops (with average diameter increase of only $1 \mu \mathrm{m}$ in 14 hours) and some of the surfaces of CSs become quite rough. In this stage, $\Delta G_{s}$ becomes very low due to the low solution concentration. Meanwhile, $\Delta G_{c}$ of CSs is greatly reduced as the sizes of CSs 


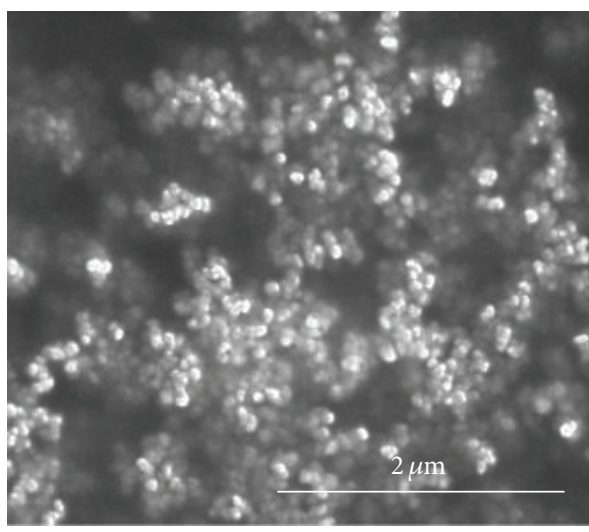

(a)

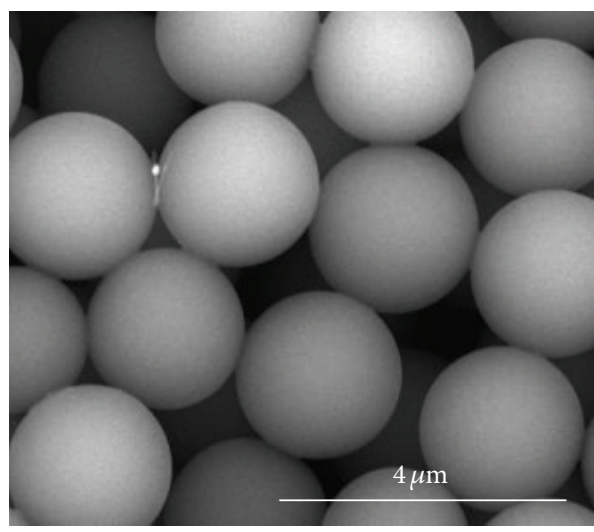

(c)

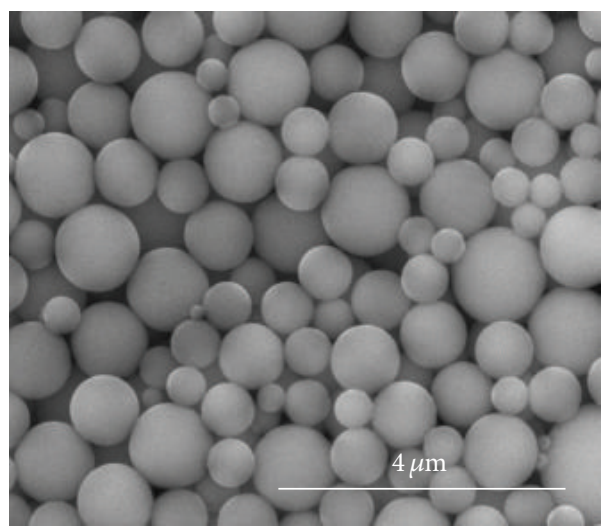

(b)

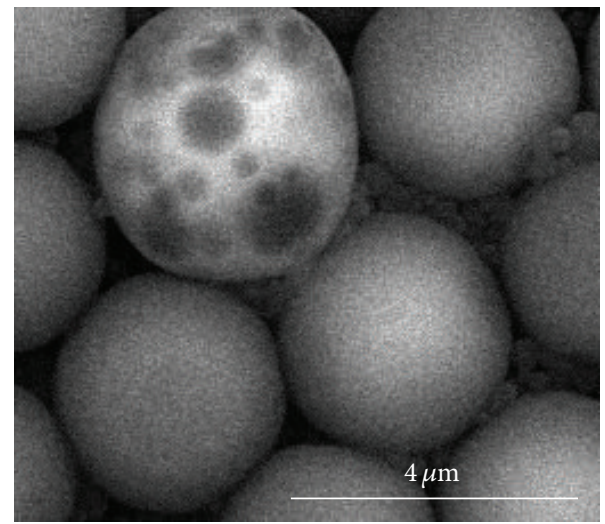

(d)

FIGURE 1: SEM images of four growth stages of CSs: reaction times (a) $5 \mathrm{~h}$, (b) $6 \mathrm{~h}$, (c) $9 \mathrm{~h}$, and (d) $22 \mathrm{~h}$.

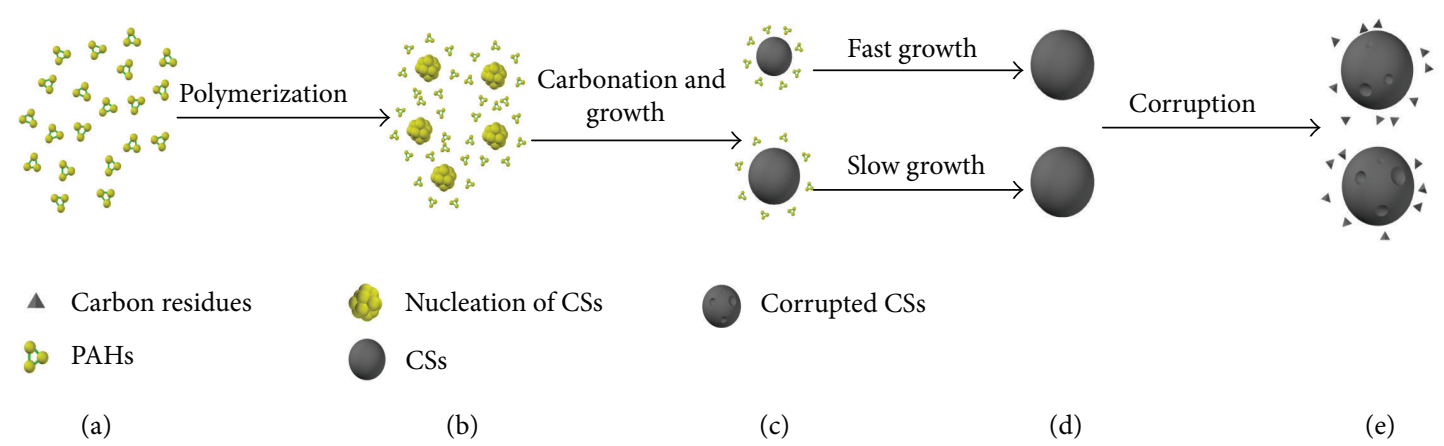

FIGURE 2: Schematic diagrams of the growth process of CSs.

grow larger. $\Delta G_{s}$ and $\Delta G_{c}$ nearly reach equilibrium during this stage. Thus, the growth of the CSs is very minimal and the average diameter of CSs only slightly increases during this stage. Meanwhile, the energy fluctuation [33] in the solution becomes the most important parameter when the energy of CSs and solution reaches equilibrium. The energy fluctuation can lead to both the absorption and desorption of carbon atoms on the surface of CSs. Carbon atoms dissolved from CSs can reform CSs, which are observed in Figure 3(d).
Therefore, surface corruption has been found on some CSs and there are a lot of irregular carbon residues around the CSs.

Through the above analysis, we found that the evolution of CSs depends on the solution free energy and the Gibbs free energy of CSs which significantly changed with the reaction time. The energy fluctuation of solution can also have a great influence on the morphologies of CSs when the energy of CSs and solution reaches equilibrium. Therefore, the size 


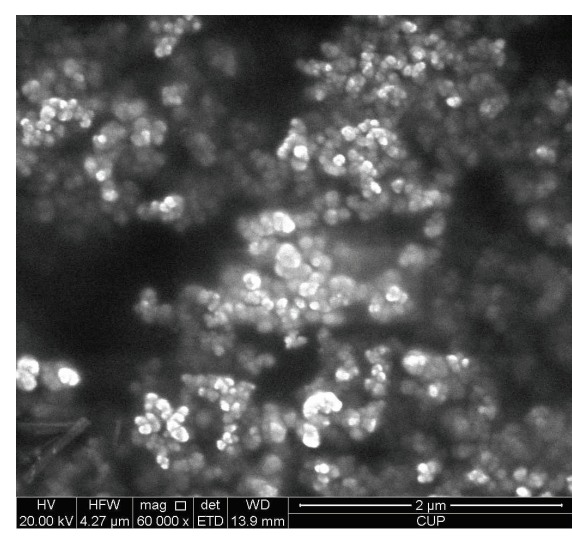

(a)

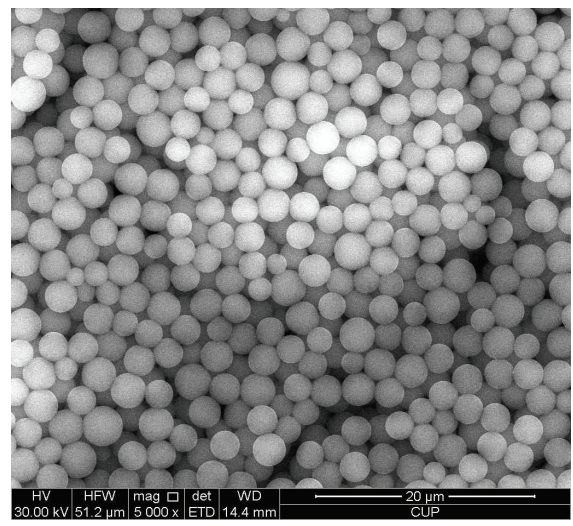

(c)

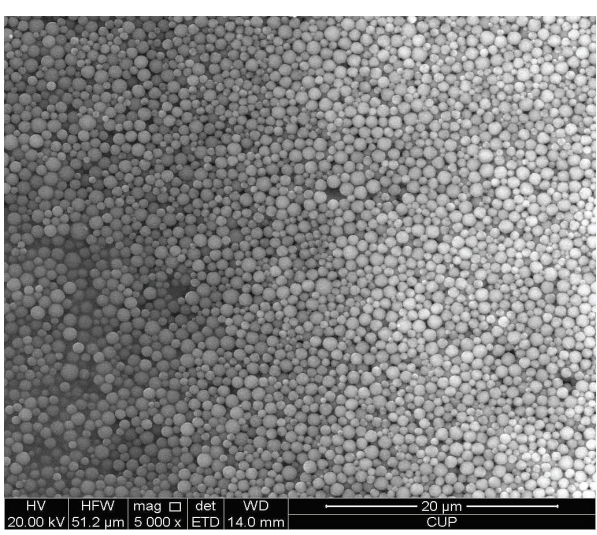

(b)

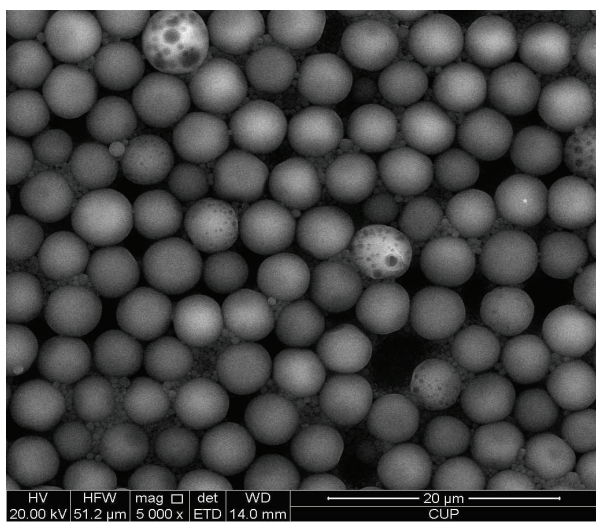

(d)

FIGURE 3: SEM images corresponding to the schematic diagrams of the growth process of CSs: (a) PAHs and nucleation of CSs, (b) different size CSs, (c) uniform size CSs, and (d) corrupted CSs.

distribution of CSs can be controlled by simply tuning the reaction time. Furthermore, CSs with porous surfaces can also be easily obtained by extending the reaction time.

\section{Conclusions}

In summary, the mechanism of the evolution process of CSs has been studied through hydrothermal method. The CSs growth process was divided into four stages (nucleation, carbonization, isokinetic growth, and corruption) to understand the formation of CSs well. Based on the nuclei theory, the changes of the size distribution and the surface morphology of the CSs have been well explained. This work not only provides deep insight into the growth mechanism of CSs, but also offers a possible way for the size and surface morphology control of CSs.

\section{Conflict of Interests}

The authors declare that there is no conflict of interests regarding the publication of this paper.

\section{Acknowledgments}

This work is supported partially by the National High-Tech R\&D Program of China (863 Program, no. 2015AA034601), the National Natural Science Foundation of China (Grants nos. 91333122, 51402106, 51372082, 51172069, 61204064, and 51202067), Ph.D. Programs Foundation of Ministry of Education of China (Grants nos. 20120036120006, 20130036110012), Par-Eu Scholars Program, and the Fundamental Research Funds for the Central Universities.

\section{References}

[1] A. J. Romero-Anaya, M. Ouzzine, M. A. Lillo-Ródenas, and A. Linares-Solano, "Spherical carbons: synthesis, characterization and activation processes," Carbon, vol. 68, pp. 296-307, 2014.

[2] Q. Qian, S. Shao, F. Yan, and G. Yuan, "Direct removal of trace ionic iodide from acetic acid via porous carbon spheres," Journal of Colloid and Interface Science, vol. 328, no. 2, pp. 257-262, 2008.

[3] D. Ugarte, "Curling and closure of graphitic networks under electron-beam irradiation," Nature, vol. 359, no. 6397, pp. 707709, 1992.

[4] W. A. de Heer and D. Ugarte, "Carbon onions produced by heat treatment of carbon soot and their relation to the $217.5 \mathrm{~nm}$ interstellar absorption feature," Chemical Physics Letters, vol. 207, no. 4-6, pp. 480-486, 1993.

[5] Z. C. Kang and Z. L. Wang, "Mixed-valent oxide-catalytic carbonization for synthesis of monodispersednano sized carbon 
spheres," Philosophical Magazine Part B, vol. 73, pp. 905-929, 1996.

[6] T. Stöckli, J.-M. Bonard, A. Châtelain, Z. L. Wang, and P. Stadelmann, "Plasmon excitations in graphitic carbon spheres," Physical Review B-Condensed Matter and Materials Physics, vol. 57, no. 24, pp. 15599-15612, 1998.

[7] C. Zhang, H. B. Wu, C. Yuan, Z. P. Guo, and X. W. Lou, "Confining sulfur in double-shelled hollow carbon spheres for lithium-sulfur batteries," Angewandte Chemie-International Edition, vol. 51, no. 38, pp. 9592-9595, 2012.

[8] X. G. Liu, S. W. Or, C. Jin, Y. H. Lv, C. Feng, and Y. P. Sun, "NiO/C nanocapsules with onion-like carbon shell as anode material for lithium ion batteries," Carbon, vol. 60, pp. 215-220, 2013.

[9] K. Zhang, Q. Zhao, Z. L. Tao, and J. Chen, "Composite of sulfur impregnated in porous hollow carbon spheres as the cathode of Li-S batteries with high performance," Nano Research, vol. 6, no. 1, pp. 38-46, 2013.

[10] M.-S. Park, J.-S. Yu, K. J. Kim et al., "Porous carbon spheres as a functional conducting framework for use in lithium-sulfur batteries," RSC Advances, vol. 3, no. 29, pp. 11774-11781, 2013.

[11] P. Trogadas, T. F. Fuller, and P. Strasser, "Carbon as catalyst and support for electrochemical energy conversion," Carbon, vol. 75, pp. 5-42, 2014.

[12] Y. Yang, K. Chiang, and N. Burke, "Porous carbon-supported catalysts for energy and environmental applications: a short review," Catalysis Today, vol. 178, no. 1, pp. 197-205, 2011.

[13] J. H. Bang, "Hollow graphitic carbon spheres for Pt electrocatalyst support in direct methanol fuel cell," Electrochimica Acta, vol. 56, no. 24, pp. 8674-8679, 2011.

[14] C. H. You, S. J. Liao, H. L. Li et al., "Uniform nitrogen and sulfur co-doped carbon nanospheres as catalysts for the oxygen reduction reaction," Carbon, vol. 69, pp. 294-301, 2014.

[15] Z. Liu, J. T. Robinson, S. M. Tabakman, K. Yang, and H. Dai, "Carbon materials for drug delivery \& cancer therapy," Materials Today, vol. 14, no. 7-8, pp. 316-323, 2011.

[16] D. Saha, K. E. Warren, and A. K. Naskar, "Soft-templated mesoporous carbons as potential materials for oral drug delivery," Carbon, vol. 71, pp. 47-57, 2014.

[17] J. A. Hubbell and A. Chilkoti, "Nanomaterials for drug delivery," Science, vol. 337, no. 6092, pp. 303-305, 2012.

[18] A. C. L. Tang, G.-L. Hwang, S.-J. Tsai et al., "Biosafety of nonsurface modified carbon nanocapsules as a potential alternative to carbon nanotubes for drug delivery purposes," PLoS ONE, vol. 7, no. 3, Article ID e32893, 2012.

[19] R. Ryoo, S. H. Joo, and S. Jun, "Synthesis of highly ordered carbon molecular sieves via template-mediated structural transformation," The Journal of Physical Chemistry B, vol. 103, no. 37, pp. 7743-7746, 1999.

[20] J. B. Joo, P. Kim, W. Kim, J. Kim, N. D. Kim, and J. Yi, "Simple preparation of hollow carbon sphere via templating method," Current Applied Physics, vol. 8, no. 6, pp. 814-817, 2008.

[21] Y. Z. Jin, C. Gao, W. K. Hsu et al., "Large-scale synthesis and characterization of carbon spheres prepared by direct pyrolysis of hydrocarbons," Carbon, vol. 43, no. 9, pp. 1944-1953, 2005.

[22] B. Friedel and S. Greulich-Weber, "Preparation of monodisperse, submicrometer carbon spheres by pyrolysis of melamineformaldehyde resin," Small, vol. 2, no. 7, pp. 859-863, 2006.

[23] C.-M. Lei, W.-L. Yuan, H.-C. Huang, S.-W. Ho, and C.-J. Su, "Synthesis and conductivity measurement of carbon spheres by catalytic CVD using non-magnetic metal complexes," Synthetic Metals, vol. 161, no. 15-16, pp. 1590-1595, 2011.
[24] H.-S. Qian, F.-M. Han, B. Zhang, Y.-C. Guo, J. Yue, and B.-X. Peng, "Non-catalytic CVD preparation of carbon spheres with a specific size," Carbon, vol. 42, no. 4, pp. 761-766, 2004.

[25] N. Baccile, G. Laurent, F. Babonneau, F. Fayon, M.-M. Titirici, and M. Antonietti, "Structural characterization of hydrothermal carbon spheres by advanced solid-state MAS 13C NMR investigations," The Journal of Physical Chemistry C, vol. 113, no. 22, pp. 9644-9654, 2009.

[26] C. Yao, Y. Shin, L.-Q. Wang et al., "Hydrothermal dehydration of aqueous fructose solutions in a closed system," Journal of Physical Chemistry C, vol. 111, no. 42, pp. 15141-15145, 2007.

[27] M. Li, W. Li, and S. Liu, "Hydrothermal synthesis, characterization, and $\mathrm{KOH}$ activation of carbon spheres from glucose," Carbohydrate Research, vol. 346, no. 8, pp. 999-1004, 2011.

[28] N. Brun, K. Sakaushi, L. Yu, L. Giebeler, J. Eckert, and M. M. Titirici, "Hydrothermal carbon-based nanostructured hollow spheres as electrode materials for high-power lithium-sulfur batteries," Physical Chemistry Chemical Physics, vol. 15, no. 16, pp. 6080-6087, 2013.

[29] V. I. Kalikmanov, "Classical nucleation theory," in Nucleation Theory, vol. 860 of Lecture Notes in Physics, pp. 17-41, Springer, Berlin, Germany, 2013.

[30] M. Li, W. Li, and S. X. Liu, "Control of the morphology and chemical properties of carbon spheres prepared from glucose by a hydrothermal method," Journal of Materials Research, vol. 27, no. 8, pp. 1117-1123, 2012.

[31] D. Kavitha and C. Namasivayam, "Experimental and kinetic studies on methylene blue adsorption by coir pith carbon," Bioresource Technology, vol. 98, no. 1, pp. 14-21, 2007.

[32] V. K. La Mer, "Nucleation in phase transitions," Industrial \& Engineering Chemistry, vol. 44, no. 6, pp. 1270-1277, 1952.

[33] G. Q. Ren, Z. Lin, B. Gilbert, J. Zhang, F. Huang, and J. K. Liang, "Evolution of $\mathrm{ZnS}$ nanostructure morphology under interfacial free-energy control," Chemistry of Materials, vol. 20, no. 7, pp. 2438-2443, 2008. 

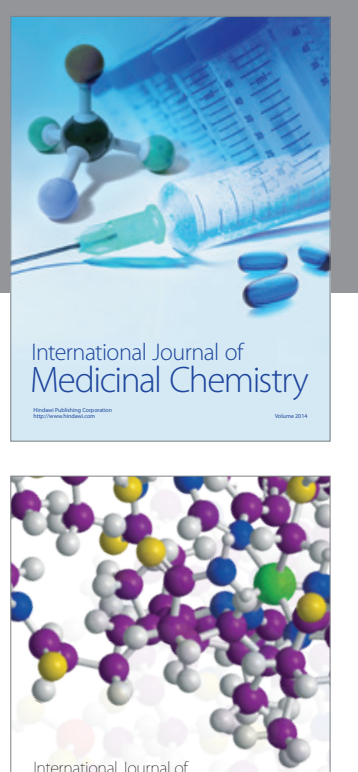

Carbohydrate Chemistry

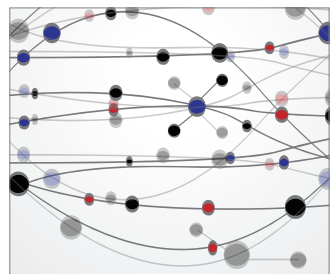

The Scientific World Journal
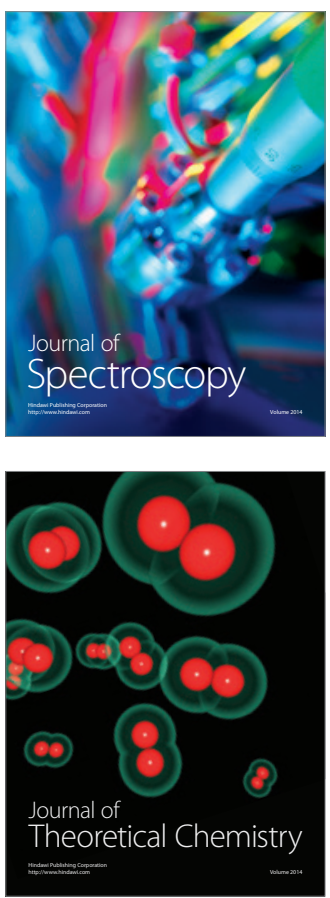
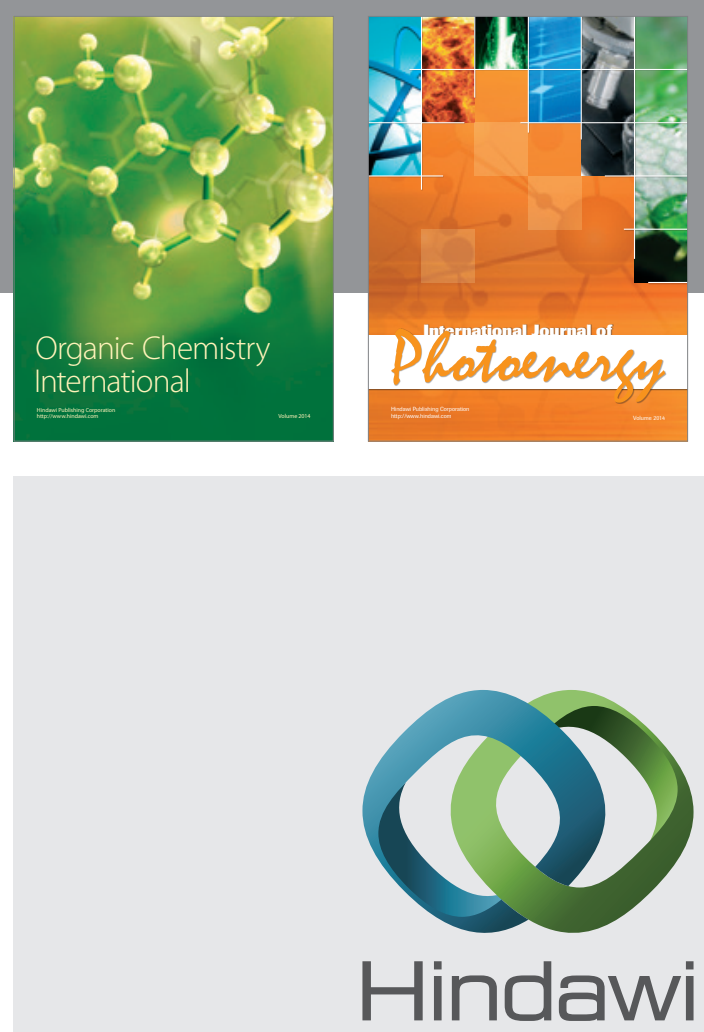

Submit your manuscripts at

http://www.hindawi.com

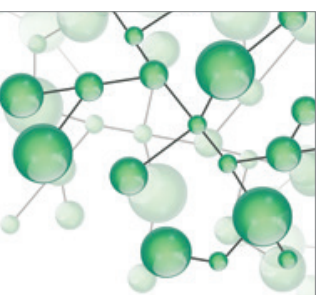

International Journal of

Inorganic Chemistry

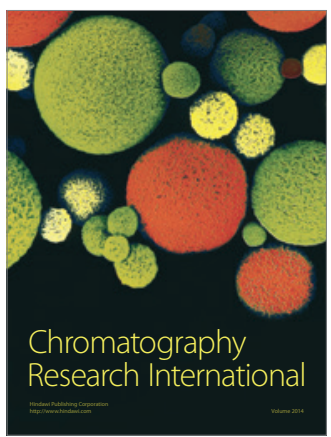

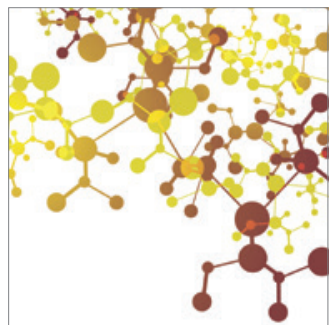

Applied Chemistry
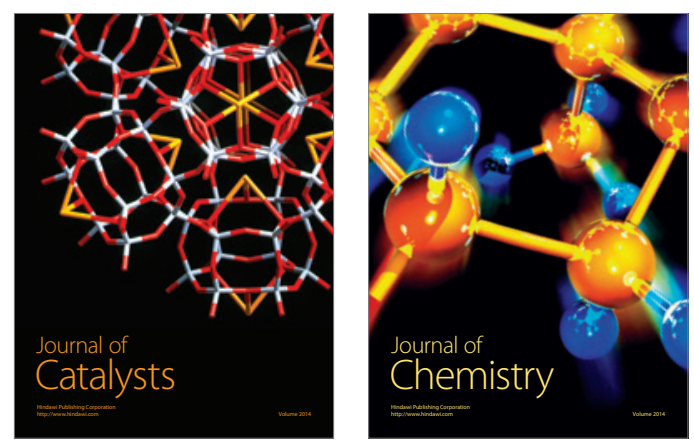
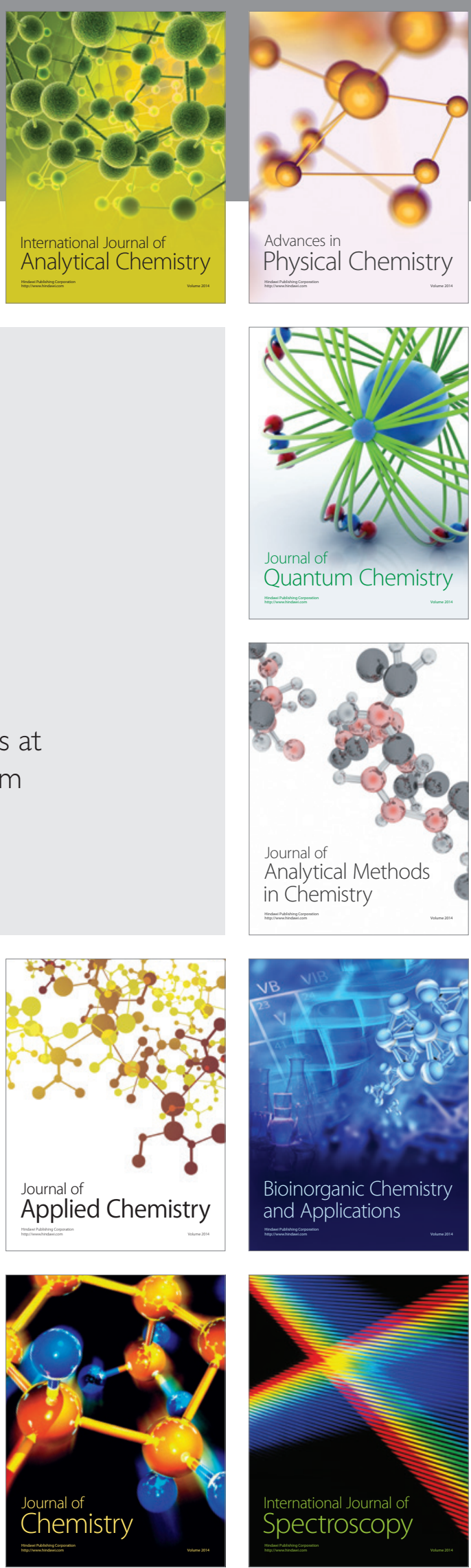